\title{
Improved Stretchable Electronics Technology for Large Area Applications
}

\author{
Frederick Bossuyt ${ }^{1}$, Thomas Vervust ${ }^{1}$, Fabrice Axisa ${ }^{1}$ and Jan Vanfleteren ${ }^{1}$ \\ ${ }^{1}$ UGent/CMST/IMEC, Zwijnaarde, Belgium.
}

\begin{abstract}
A novel technology for stretchable electronics is presented which can be used for the realization of wearable textile electronics and biomedical implants. It consists of rigid or flexible component islands interconnected with stretchable meander-shaped copper conductors embedded in a stretchable polymer, e.g. PDMS. The technology uses standard PCB manufacturing steps and liquid injection molding techniques to achieve a robust and reliable product. Due to the stretchable feature of the device, conductors and component islands should be able to withstand a certain degree of stress to guarantee the functionality of the system. Although the copper conductors are meander-shaped in order to minimize the local plastic strain, the lifetime of the system is still limited by the occurrence of crack propagation through the copper, compromising the connectivity between the functional islands. In order to improve the lifetime of the conductors, the most important feature of the presented technology is the use of spin-on polyimide as a mechanical support for the stretchable interconnections and the functional flexible islands. In this way, every stretchable copper connection is supported by a $20 \mu \mathrm{m}$ layer of polyimide being shaped in the same manner as the above laying conductor. The grouped SMD components and straight copper tracks on the functional islands are also supported by a complete $20 \mu \mathrm{m}$ polyimide layer. By use of the polyimide, the reliability of the stretchable interconnections, the straight interconnections on the flexible islands and the transitions between the stretchable and non-stretchable parts is improved. This approach results in a significant increase of the lifetime of the stretchable interconnections as it is doubled. In this contribution, the different process steps and materials of the technology will be highlighted. Initial reliability results will be discussed and the realization of some functional demonstrators containing a whole range of different components will further illustrate the feasibility of this technology. The advantages and disadvantages in terms of processability, cost and mechanical strength of the photo-definable polyimide will be covered.
\end{abstract}

\section{INTRODUCTION}

In our stretchable molded interconnect technology (SMI-technology) a temporary carrier with wax is used to hold metal tracks and components in place during processing. In the end, the electronic system is molded in a stretchable material by molding the top part, removing the temporary carrier and molding the back part. In this way, a stretchable electronic system is obtained. Components are connected with each other with stretchable copper tracks both embedded in a stretchable material [1-2]. Reliability tests on such stretchable electronic systems [3] have driven us to improve this technology in order to improve the mechanical reliability of the stretchable interconnections and the connections between the components on the functional islands. In this paper, an improved technology is presented based on the use of a photodefinable polyimide. The polyimide will remain in the final stretchable electronic system, supporting the metallic stretchable interconnections and places where rigid components are soldered. The principle is shown in Fig. 1. Without the polyimide stress on the straight connections and on the 
solder connections will lead to breaking of the electronic system during stretching. The use of the flexible, non-stretchable polyimide will shift the stretching from the straight connections and the solder connections to the border of the component island, where measures are taken to cope with the stress.

A

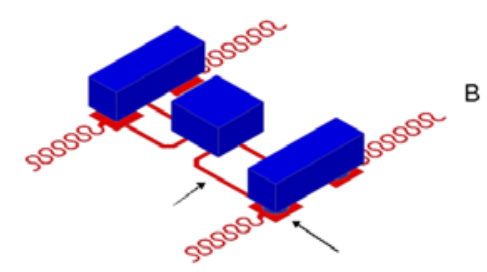

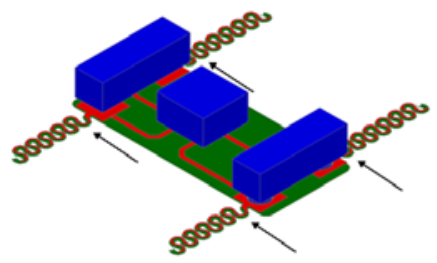

Fig. 1. Stretchable electronic system without (A) and with (B) the use of a polyimide supporting layer on places with components and conductive meanders

In this technology, it's possible to make a fluent transition from stretchable parts to nonstretchable parts, thus between the rigid component islands and the stretchable connectors by shaping the polyimide and the copper.

\section{EXPERIMENT}

\section{Process description}

The process flow is shown in Figure 2 and can be shortly described as follows:

[1] A copper sheet is used as starting point for processing.

[2] A flexible supporting/isolation material is patterned on the copper sheet by a photolithographic process. Photodefinable polyimide (HD4100, HD Microsystems) is used for this purpose. The flexible material is used as support for the meandered conductors defined in the copper and support for the flexible functional islands where straight conductor tracks are defined and SMD components are mounted.

[3] The substrate is laminated by use of temporary glue (wax, Quickstick 135, South Bay Technology Inc.) on a temporary rigid/flexible carrier with the side of the copper on top, in order to be able to pattern the copper sheet by wet-etching it. In this way, the copper meandered conductors are defined and the tracks on the functional flexible islands are defined.

[5] Soldermask is applied, a copper finish is applied (OSP), components are mounted by reflow soldering using SAC.

[6] The top part is molded using an elastomer (Sylgard 186, Dow Corning) and an adhesion promoter OS1200 (Dow Corning)

[7] The temporary carrier is removed; the temporary glue (Quickstick 135 wax) is removed [8] The back part is molded using the same elastomer (Sylgard 186) and an adhesion promoter OS1200

[9] We end up with a completely embedded stretchable electronic circuit. 

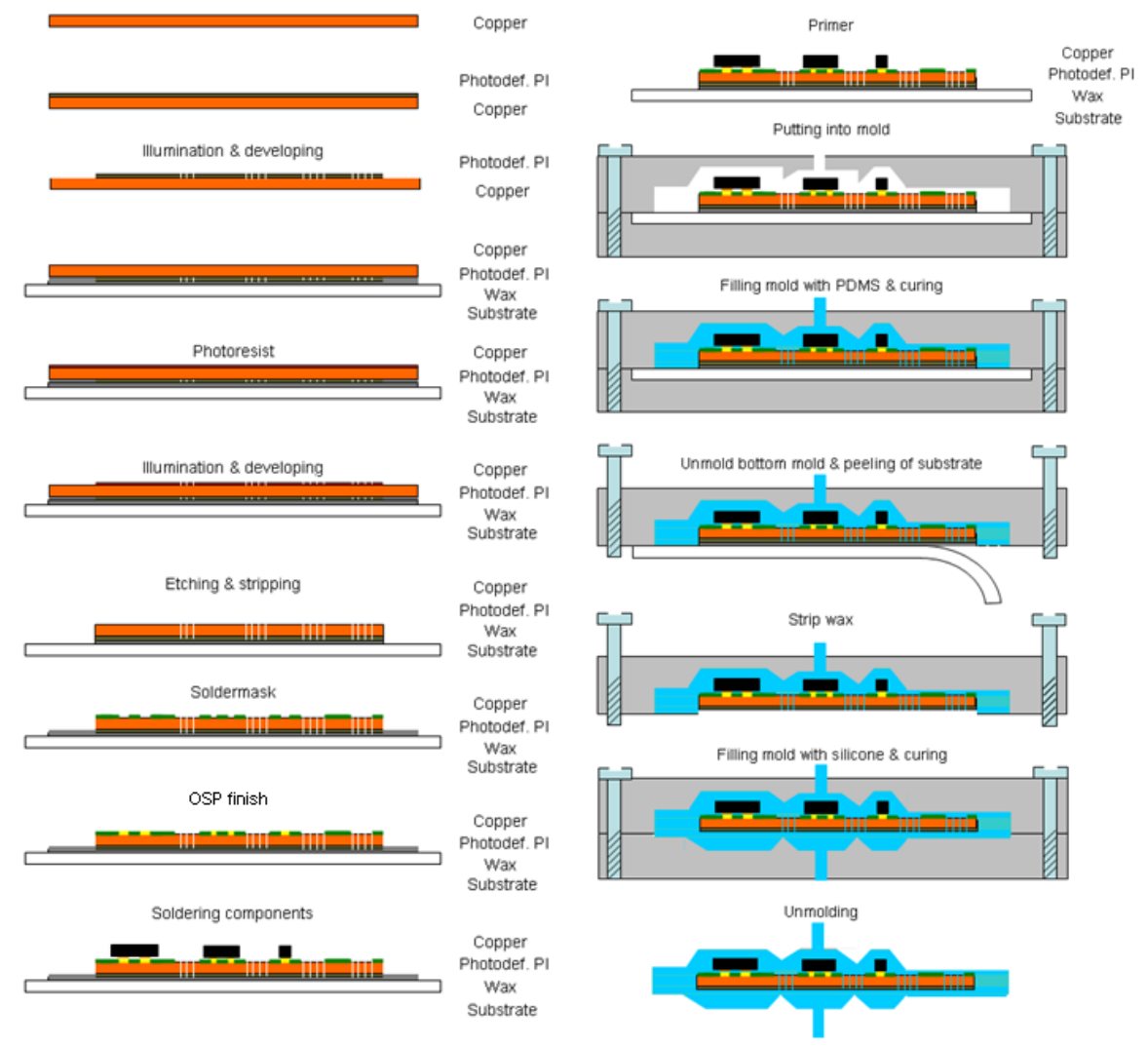

Fig. 2. Process flow

To show the feasibility of this process flow, we used an electronic design, adopted in a layout for our stretchable electronics technology. The 3 different layers are shown in Fig. 3a.

It consists of 3 flexible islands connected with each other through stretchable interconnections, having a width of $100 \mu \mathrm{m}$. The islands and stretchable interconnections are supported by the photodefined polyimide layer. On places where components are, soldermask is foreseen.

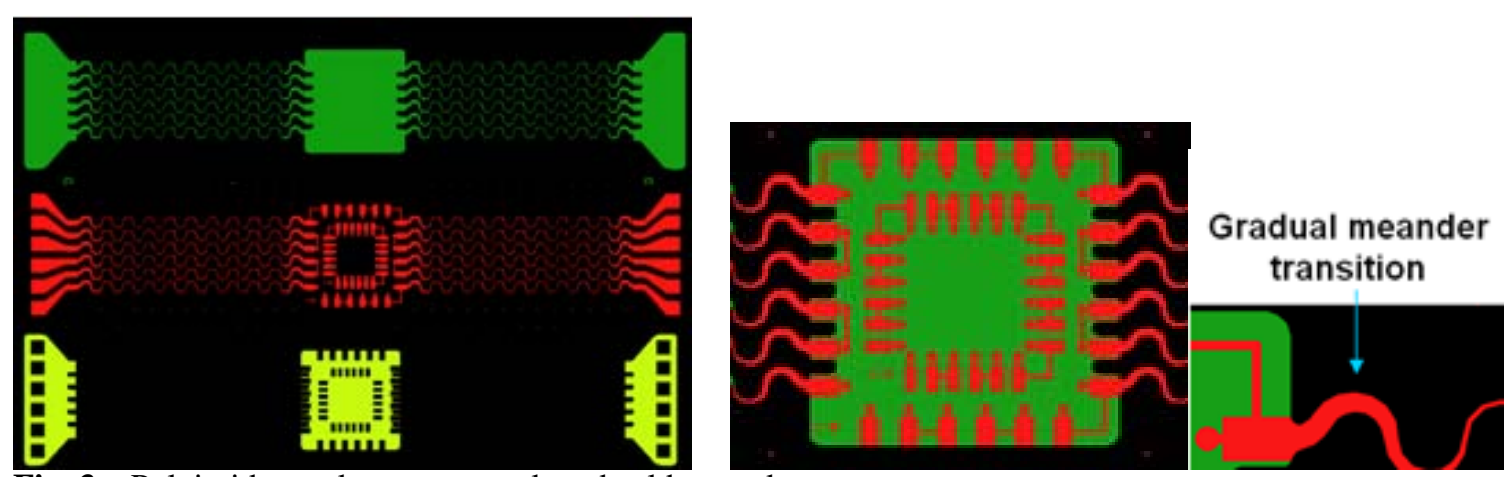

Fig. 3a. Polyimide mask, copper mask and solder mask

Fig. 3b. Gradual meander transition at flex/stretch interface

The design has been optimized in order to cope with stresses coming up during mechanical stretching of the embedded electronic system. By having the possibility of shaping the supporting polyimide, a better transition from the rigid (components, interposers,..) parts to the 
stretchable parts is possible.

The patterning of the polyimide for supporting the conductive meanders doesn't have to be the same pattern as the meanders itself; it can be optimized in order to increase the connection reliability. Flex-stretch interface measures have been taken and are shown in Fig. 3b.

In Fig. 4a, one of the samples is shown where the HD4100 polyimide layer is patterned on. The alignment marks can be seen in the four corners of the sample, giving us the opportunity to laser drill holes through the polyimide-copper. These samples are covered on the polyimide side with the wax layer and laminated on the wax covered carrier. After lamination, patterning of the copper is done where the copper layer is aligned by use of the laser drilled holes.

In Fig. 4b, the samples are shown after copper etching, resulting in a substrate-wax-polyimidecopper stack. Some more detailed pictures are shown in Fig. 4c.
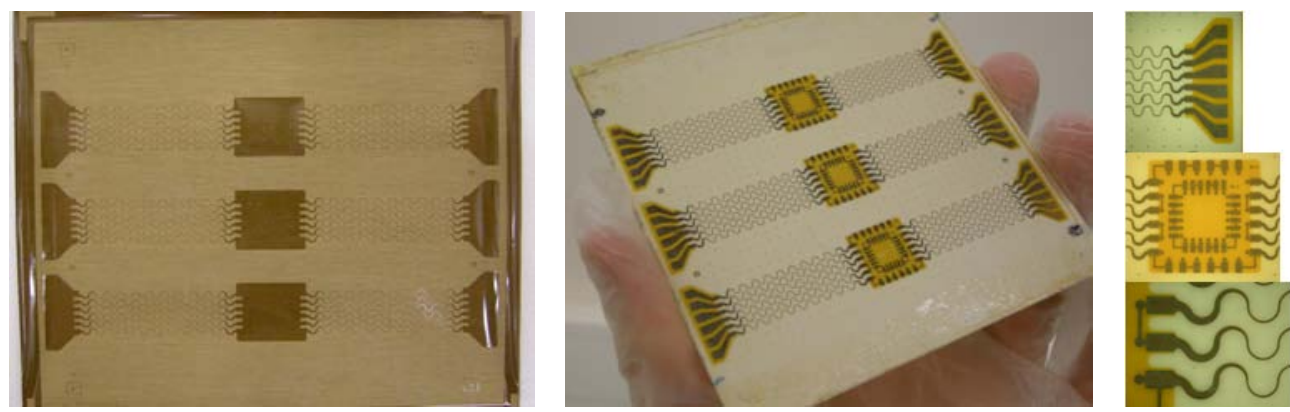

Fig. 4a. Substrate with polyimide-copper stack

Fig. 4b. Substrate with polyimide-copper stack on temporary carrier after copper etching

Fig. 4c. Substrate with polyimide-copper stack on temporary carrier after copper etching: closer view

In Fig. 5a, the samples are shown after application of soldermask.
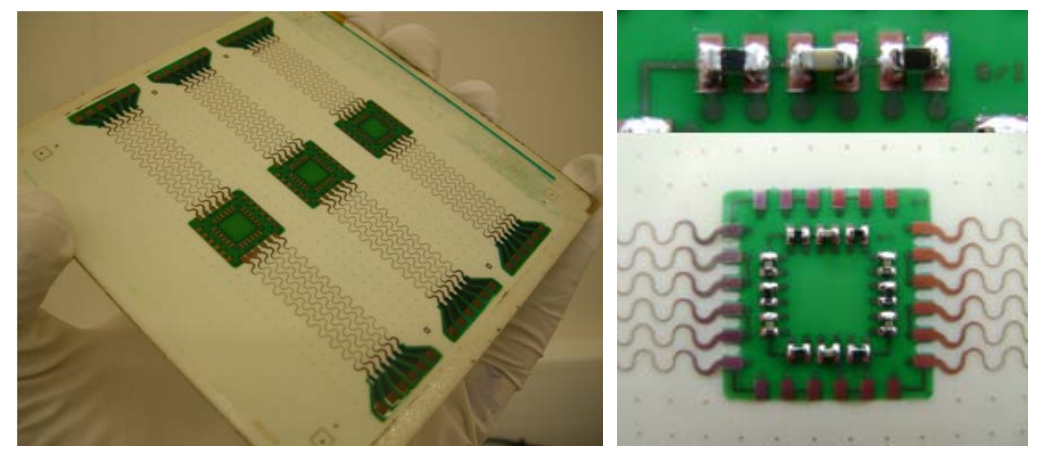

Fig. 5a. Substrate with polyimide-copper stack covered with soldermask

Fig. 5b. Substrate with polyimide-copper stack covered with soldermask and SMD components soldered

Before soldering components, the copper is cleaned and protected from oxidation by OSP (MecSeal, Centurion Specialty Chemicals). The result from reflow soldering some SMD components (0402 capacitors and resistors) on the flexible island is shown in Fig. 5b. The final steps are the transfer of the electronic circuit into a stretchable polymer. We put adhesion promoter OS1200 primer (Dow Corning) on the substrate and use Sylgard 186 (Dow Corning) as embedding material. The substrate is placed into the mold and then, silicone is injected into the mold, leading to the embedding of the top part of the circuit. The PDMS is cured at $50^{\circ} \mathrm{C}$ to make sure the wax doesn't melt. For higher curing temperatures, the wax melts, mixes up with PDMS and results in an uncurable wax-PDMS mixture. The curing is done for at least 4 hours. The bottom part of the mold is then removed. The flexible substrate is also 
removed by heating up the mold into an oven at $200^{\circ} \mathrm{C}$. In this way, the wax melts and the temporary substrate is easily removable. The wax is removed by use of an acetone and water rinse. The result of the molding is shown in Fig. 6. A view on the molding showing the fluent transitions between thinner silicone parts and thicker silicone parts is shown.

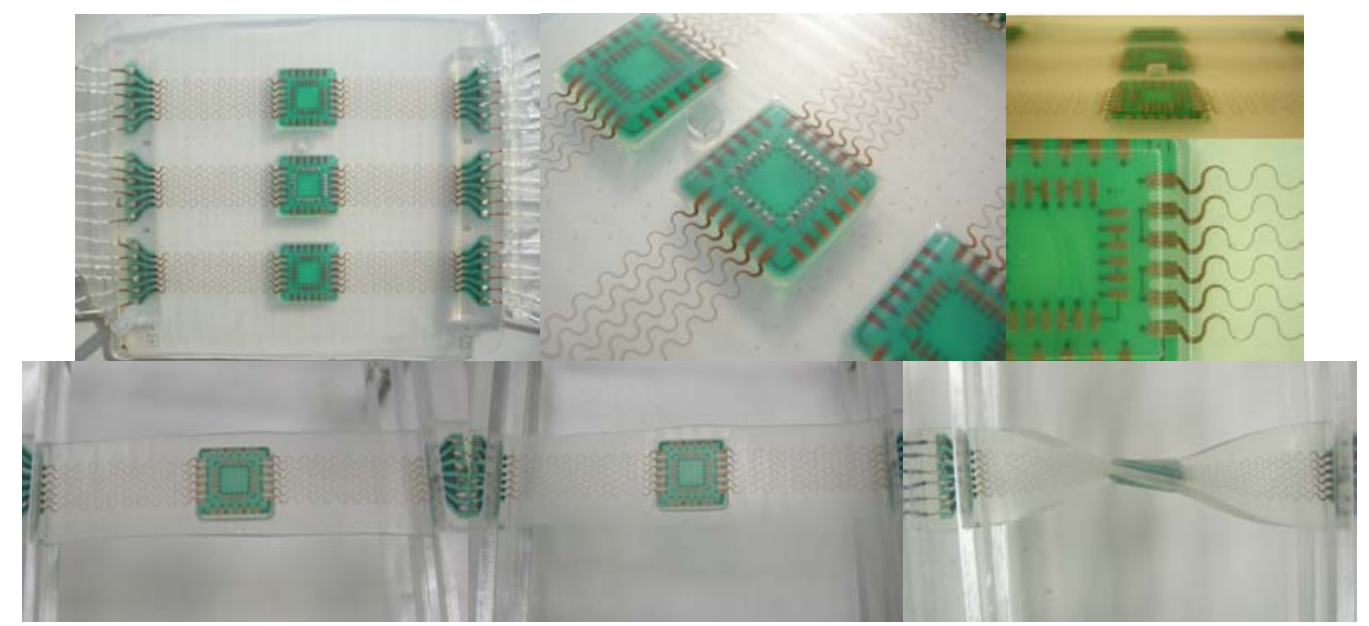

Fig. 6. (Top) Stretchable system completely embedded in Sylgard 186 - (Bottom) Stretchable system completely embedded in Sylgard 186 in non-stretched form, in 10\% stretched form and in twisted form

The thicker silicone parts are applied in regions where flexible islands are and components are mounted. The thinner silicone parts are present in the regions where meanders are defined. In this way, we make stretching more easily in the thinner parts where the conductors are especially designed to be stretchable. In the thicker parts, consisting of non-stretchable components, the stretching will be none or much lower. In Fig. 6, a non-stretched sample, a 10\% sample and a twisted sample is shown. In Fig. 7, the dimensions of the molded sample are shown.

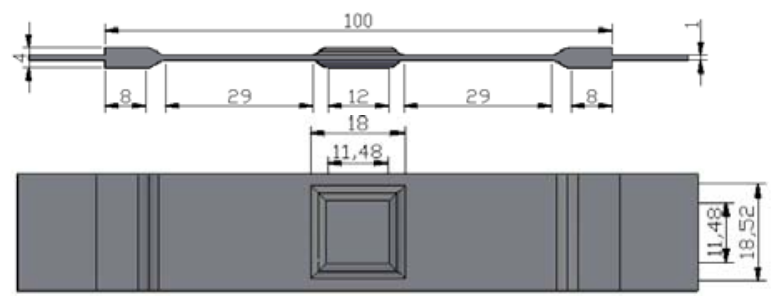

Fig. 7. Dimensions (in mm) of molded sample

\section{DISCUSSION}

Reliability experiments have shown that the use of this polyimide as support for the stretchable interconnections doubles the cyclic lifetime of the copper interconnects [3]. To give an indication of the lifetime of the sample presented, cyclic strain tests have been performed, leading to an average of 2500 cycles for an elongation of $10 \%$ and a strain rate of $1 \% / \mathrm{sec}$.

The used photodefinable polyimide is manufactured for use as insulation and soldermask on dies, where accurate definition and fine pitch are needed. The photodefinable polyimide which is expensive and too good in terms of possible feature sizes - which we don't need for our application - has being used because of the good mechanical properties of polyimide and of course for its photodefinable property. There are other, cheaper ways to apply polyimide. This technology has already been used for the realization of some demonstrators. In Fig. 8, a fitness activity monitor is shown (Philips design), consisting of five 4-layer electronic interposer 
boards including a rechargeable battery, a wireless circuit and antenna, accelerometers,.. which are mounted on a polyimide supported SMI substrate and completely embedded in PDMS.

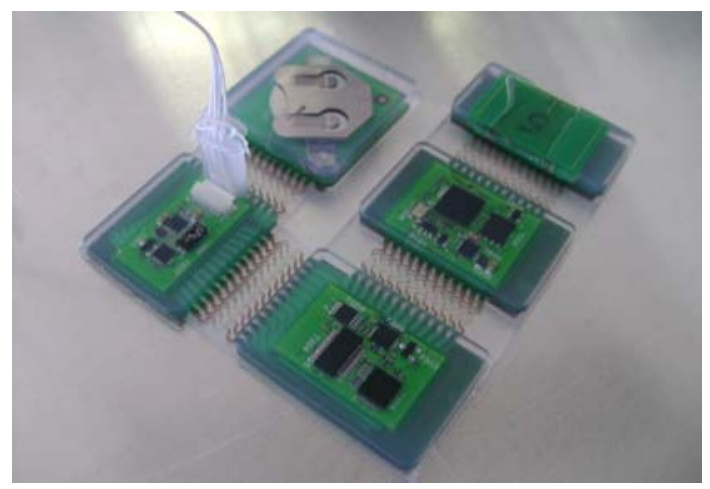

Fig. 8. Philips fitness activity monitor

\section{CONCLUSIONS}

We have developed an improved version of our low-cost stretchable electronics technology, which is based on standard PCB manufacturing technologies. The use of polyimide as mechanical support for the stretchable conductors and flexible component islands is a major improvement in terms of mechanical reliability of the stretchable electronic system. The photodefinable feature of the used polyimide allows us to define the supporting structures. In this way, more fluent transitions between rigid, flexible and stretchable parts can be defined, shifting the stresses to areas where measures are taken to cope with them. Reliability experiments have shown us that the lifetime of the stretchable interconnections is improved by a factor of 2 by the use of the polyimide. By molding in PDMS, reliability improving measures can also be taken by defining thicker and thinner parts and having smooth transitions of polymer thickness between these parts. A complex functional demonstrator has been made in this technology.

\section{ACKNOWLEDGMENTS}

This work is supported by European Commission Research programme STELLA (contract number 028026). The work is also supported by the Belgian Science Policy (Belspo) through the Sweet project (contract number P2/00/08).

\section{REFERENCES}

1. D. Kim, J. Xiao, J. Song, Y. Huang, J. A. Rogers. Stretchable, Curvilinear Electronics Based on Inorganic Materials, Adv. Mater. 2010, 22, 1-17

2. D. Brosteaux, F. Axisa, M. Gonzalez, and J. Vanfleteren, Design and Fabrication of Elastic Interconnections for Stretchable Electronic Circuits, IEEE Electron Device Letters, vol. 28, issue 7, pp. 552-554

3. M. Gonzalez, F. Axisa, M. Vanden Bulcke, D. Brosteaux, B. Vandevelde, J. Vanfleteren, Design of metal interconnects for stretchable electronic circuits, Microelectronics Reliability 48 (2008) 825-832

4. T. Sterken, F. Bossuyt, R. Verplancke, T. Vervust, F.Axisa, J. Vanfleteren. Lifetime of Stretchable Meander-Shaped Copper Conductors in PDMS Subjected to Cyclic Elongation, 
Proceedings of MRS Spring meeting 2010, Symposium JJ, (San Francisco, USA), pp. -, April 2010. 